\title{
Neues Denken für die Wirtschaft von morgen
}

\author{
Die globalen Klimastreiks und die Corona-Pandemie erzeugen \\ Druck zur Transformation der Wirtschaft. Economists4future \\ können ihn in neue Dialogformen, kreative Vorstellungskräfte \\ und friedlichen Gestaltungswillen wandeln. \\ Von Lars Hochmann und Silja Graupe
}

D ie gegenwärtigen Probleme sind gewaltig. Wir Menschen stehen vor den Aufgaben, die Erderwärmung unter $1,5^{\circ} \mathrm{C}$ zu halten, die massenhafte Auslöschung von Tierarten und Pflanzensorten $\mathrm{zu}$ stoppen und stattdessen Vielfalt zu fördern, die globale Pandemieanfälligkeit $\mathrm{zu}$ beseitigen und dabei globale wie lokale Unterdrückung und Ungerechtigkeit aufzulösen sowie Solidarität und Teilhabe zu stärken. Doch allein Einsichten in diese Probleme zu gewinnen und sich auf basale Fakten zu einigen, erweist sich als schwierig. Ungleich schwieriger ist, wie sie friedlich und konsequent angegangen werden können. Wissenschaft sollte auch um die Befähigung willen da sein, inmitten von Unsicherheit und Komplexität dialogisch-visionäre Schaffenskräfte zu bilden.

\section{Wende zum Neuen und Möglichen}

Die Ernährungs-, Energie-, Wohnoder Verkehrswenden zeigen dabei, dass es kaum einen Bereich gesellschaftlicher Versorgung gibt, der nicht neu gestaltet werden muss, um die Lebensgrundlagen auf diesem Planeten zu schützen und zu erhalten - im Norden wie im Süden, heute wie morgen. Für diese Jahrhundertaufgaben braucht es ökonomischen Bildungs- und Ideenreichtum, Vorstellungskräfte und Gestaltungswillen, um Wirtschaft neu zu denken und abseits ausgetrampelter Pfade echten Wandel reflektiert und verantwortungsvoll auf den Weg zu bringen.
All dies ist lange bekannt. Neu hingegen ist der öffentliche Druck, auch wirklich etwas zu ändern. Die vielen, vor allem jungen Menschen von Fridays for Future schaffen ihn, indem sie die Beweislast zunehmend umkehren. Bis zuletzt mussten sich plural und andersdenkende Ökonom/innen für ihr Interesse an Gemeinsinn und Nachhaltigkeit fachlich wie öffentlich rechtfertigen, während der Mainstream oftmals wie selbstverständlich oder gar blindlings Partei ergreifen konnte für die Interessen einer Wirtschaft, die den Planeten zerstört.

Heute liegt ein Wandel in der Luft für neues ökonomisches Denken und Gestalten. Die Generation der Fridays for Future wird es nicht länger akzeptieren, wenn ihre Fragen nach Zukunftsfähigkeiten im Studium der Ökonomie nicht beantwortet, wenn die Zerstörung der Biosphäre gemäß Marginalkostenberechnung als ökonomisches Optimum bezeichnet und lediglich als Kollateralschaden abgetan oder die Vielfalt an möglichen Wirtschaftsweisen nonchalant auf geldvermittelten Opportunismus reduziert werden.

\section{Unternehmensvielfalt denkbar machen}

Dies bedeutet nicht, das Denken von gestern ad acta zu legen. Es eignet sich für eine umfassende Bestandsaufnahme mit dem Ziel, Sinnvolles zu bewahren, sowie für ein agnotologisches Verstehen, wie Un- und Falschwissen durch Manipulation, Vereinseitigung und andere
Formen epistemischer Gewalt geschaffen und verteidigt wird. Erst diese Auseinandersetzung ermöglicht die gebildete Entscheidung, welche Denkstile tradiert und welche geändert oder neu hinzugewonnen werden sollten.

Während dies Zeit braucht, sind die ökonomischen Praktiken schon heute vielfältiger und innovativer als ihr Ruf. Von solidarischer Produktion, Prosuming, Repairing oder Unternehmen in Verantwortungseigentum gibt es vielfältigen Aufbruch einer Wirtschaft, die dem Leben dient, nicht andersherum. Diese unternehmerischen Initiativen ermöglichen Teilhabe, bereiten das Neue im Alten vor und ermöglichen so eine sichere und souveräne Versorgung mit alledem, was zu einem gelingenden Leben dazugehört. Economists4future sollten ihnen Stimme und Nachdruck geben, sie reflexiv und plural fundieren sowie als kritische Dialogpartner/innen fungieren.

\section{Zukunft in gute Hände legen}

Die Wirtschaft von morgen entsteht nicht länger aus Begriffsidealen, die auswendig gelernt und dann mühselig der Praxis übergestülpt werden. Sie kommt auf den Weg als Zusammenspiel aus Reflexion und Tätigsein, das drängende Zeitfragen bewältigen hilft. Versierte Zukunftsgestalter/innen brauchen Bildungsangebote, die nachhaltig, visionär und verantwortungsstark machen. Diese Könnerschaften resultieren aus einem neuen ökonomischen Denken, das reflexiv, transparent, divers, partizipativ und befähigend ist. Dies ist die Zeit der economists4future. Gemeinsam können wir die Zukunft aller in gute Hände legen.

\section{AUTOR/INNEN + KONTAKT}

Lars Hochmann vertritt die Professur für Plurale Ökonomie und Silja Graupe ist Profes sorin für Ökonomie und Philosophie an der Cusanus Hochschule für Gesellschaftsgestaltung.

Cusanus Hochschule für Gesellschaftsgestaltung, Bahnhofstraße 5, 54470 Bernkastel-Kues. Tel.: +496531 9724257, Fax.: +4965319724258, E-Mail: lars.hochmann@cusanus-hochschule.de, Silja.graupe@cusanus-hochschule.de, Website: www.lars-hochmann.de, www.silja-graupe.de 